\title{
COVID-19: interim guidance on rehabilitation in the hospital and post-hospital phase from a European Respiratory Society- and American Thoracic Society-coordinated international task force
}

\author{
Martijn A. Spruit (10) $1,3,4$, Anne E. Holland ${ }^{5,6,7}$, Sally J. Singh ${ }^{8,9,10}$ \\ Thomy Tonia ${ }^{11}$, Kevin C. Wilson ${ }^{12}$ and Thierry Troosters ${ }^{13,14}$
}

@ERSpublications

Experts recommend identification of unmet rehabilitation needs in patients with COVID-19 who are discharged from the hospital, and consequent tailored rehabilitative interventions, accompanied by compliance with the highest biosecurity standards https://bit.ly/3geBsPE

Cite this article as: Spruit MA, Holland AE, Singh SJ, et al. COVID-19: interim guidance on rehabilitation in the hospital and post-hospital phase from a European Respiratory Society- and American Thoracic Society-coordinated international task force. Eur Respir J 2020; 56: 2002197 [https://doi.org/10.1183/ 13993003.02197-2020].

\section{ABSTRACT}

Background: Patients with coronavirus disease 2019 (COVID-19) or post-COVID-19 will probably have a need for rehabilitation during and directly after the hospitalisation. Data on safety and efficacy are lacking. Healthcare professionals cannot wait for published randomised controlled trials before they can start these rehabilitative interventions in daily clinical practice, as the number of post-COVID-19 patients increases rapidly. The Convergence of Opinion on Recommendations and Evidence process was used to make interim recommendations for rehabilitation in the hospital and post-hospital phases in COVID-19 and post-COVID-19 patients, respectively.

Methods: 93 experts were asked to fill out 13 multiple-choice questions. Agreement of directionality was tabulated for each question. $\geqslant 70 \%$ agreement on directionality was necessary to make consensus suggestions. Results: 76 (82\%) experts reached consensus on all questions based upon indirect evidence and clinical experience on the need for early rehabilitation during the hospital admission, the screening for treatable traits with rehabilitation in all patients at discharge and 6-8 weeks after discharge, and around the content of rehabilitation for these patients. It advocates for assessment of oxygen needs at discharge and more comprehensive assessment of rehabilitation needs, including physical as well as mental aspects 6-8 weeks after discharge. Based on the deficits identified, multidisciplinary rehabilitation should be offered with attention on skeletal muscle and functional as well as mental restoration.

Conclusions: This multinational task force recommends early, bedside rehabilitation for patients affected by severe COVID-19. The model of pulmonary rehabilitation may suit as a framework, particularly in a subset of patients with long-term respiratory consequences.

This article has an editorial commentary: https://doi.org/10.1183/13993003.02889-2020

This article has supplementary material available from erj.ersjournals.com

The goal of consensus guidance is to standardise care, thereby improving outcomes and facilitating research. The suggestions in this document do not constitute official positions of the American Thoracic Society, European Respiratory Society, or the institutions of the task force members. They should not be considered mandates as no suggestion can incorporate all potential clinical circumstances. The suggestions are interim guidance that should be re-evaluated as evidence accumulates.

Received: 6 June 2020 | Accepted after revision: 23 July 2020

Copyright OERS 2020. This version is distributed under the terms of the Creative Commons Attribution NonCommercial Licence 4.0. 


\section{Introduction}

Globally, millions of people are infected with the severe acute respiratory syndrome coronavirus 2 (SARS-CoV-2), causing coronavirus disease 2019 (COVID-19) [1]. A proportion of the confirmed COVID-19 patients are admitted to the hospital for acute care, due to severe respiratory symptoms and in some cases even acute respiratory distress requiring prolonged mechanical ventilation [2].

It is very likely that a proportion of the COVID-19 patients will have a need for rehabilitative interventions during and directly after the hospitalisation [3-6]. This approach is recommended by the World Health Organization [7]. However, data on safety and efficacy of rehabilitation during and/or after hospitalisation in these patients are lacking. Equally, healthcare professionals cannot wait for well-designed randomised controlled trials to be published before they can start these rehabilitative interventions in daily clinical practice, as the number of COVID-19 patients increases rapidly every day.

Recently, WILson et al. [8] used the Convergence of Opinion on Recommendations and Evidence (CORE) process to make recommendations for the management of COVID-19. The CORE process is a consensus-based approach to making clinical recommendations that has been shown to yield recommendations that are concordant with recommendations developed using Institute of Medicine-adherent methodology [9]. The same methodology was used to develop interim guidance on rehabilitative interventions in the hospital and post-hospital phase in COVID-19 patients pending empirical evidence.

\section{Methods}

An ad hoc international task force was assembled, including the European Respiratory Society (ERS) and American Thoracic Society (ATS) key opinion leaders as well as key opinion leaders and clinical experts from other relevant societies in the field of pulmonary rehabilitation. Invitations were sent to 93 experts within the field of pulmonary rehabilitation, respiratory and critical care medicine of physical medicine and rehabilitation. $76(82 \%)$ agreed to participate. As described before [8], the SurveyMonkey platform (SurveryMonkey, San Mateo, CA, USA) was used to create a 13-question multiple-choice survey. Each question consisted of three parts: 1) presentation of the question in a modified PICO (patient, intervention, comparator, outcomes) format; 2) a multiple-choice question asking for strong or conditional recommendation for or against a course of action or no recommendation; and 3) a free-text box for comments.

After publishing an initial web-based blog on April 3, 2020 and seeking global input [10], the core team of experts (MA Spruit, AE Holland, SJ Singh, T Troosters) put together a list of possible relevant questions, and during three teleconferences, consensus was reached about which question to maintain. Wording of the questions was adapted with input of ERS and ATS methodology teams to ensure unequivocal interpretation and consistency. The survey was not piloted in order to have more timely recommendations.

The survey was administered once from April 27 to May 11, 2020, including several reminders. Agreement of directionality was tabulated for each multiple-choice question. $\geqslant 70 \%$ agreement on directionality was necessary to make consensus suggestions. The proportion of respondents per choice per question was calculated and expressed as a percentage of the total number of respondents. As $>70 \%$ agreement on directionality was reached in all questions from the first round, no second round was necessary.

\footnotetext{
Affiliations: ${ }^{1}$ Dept of Research and Development, CIRO, Horn, The Netherlands. ${ }^{2}$ NUTRIM School of Nutrition and Translational Research in Metabolism, Maastricht, The Netherlands. ${ }^{3}$ Dept of Respiratory Medicine, Maastricht University Medical Centre (MUMC+), Maastricht, The Netherlands. ${ }^{4}$ REVAL - Rehabilitation Research Center, BIOMED - Biomedical Research Institute, Faculty of Rehabilitation Sciences, Hasselt University, Diepenbeek, Belgium. ${ }^{5}$ Dept of Allergy, Immunology and Respiratory Medicine, Monash University, Melbourne, Australia. ${ }^{6}$ Dept of Physiotherapy, Alfred Health, Melbourne, Australia. ${ }^{7}$ Institute for Breathing and Sleep, Melbourne, Australia. ${ }^{8}$ Dept of Respiratory Science, University of Leicester, Leicester, UK. ${ }^{9}$ Centre for Exercise and Rehabilitation Science, Biomedical Research Centre-Respiratory, University Hospitals of Leicester NHS Trust, Leicester, UK. ${ }^{10}$ Covid Advisory Group, British Thoracic Society, London, UK. ${ }^{11}$ Institute of Social and Preventive Medicine, University of Bern, Bern, Switzerland. ${ }^{12}$ Division of Allergy, Pulmonary, Critical Care, and Sleep Medicine, Boston University School of Medicine, Boston, MA, USA. ${ }^{13}$ KU Leuven, Dept of Rehabilitation Sciences, Leuven, Belgium. ${ }^{14}$ Pulmonary Rehabilitation, University Hospital Gasthuisberg, Leuven, Belgium.
}

Correspondence: Martijn A. Spruit, Dept of Research and Development, CIRO, Hornerheide 1, 6085 NM, Horn, The Netherlands. E-mail: martijnspruit@aciro-horn.nl 


\section{Results}

The international task force suggests that hospitalised patients with COVID-19 should receive rehabilitation at/around the bedside (critical care and/or ward based) until safe for discharge to the home environment

Rationale

While most people infected with the COVID-19 virus have mild disease [11], some have more severe symptoms requiring hospital admission, and $20 \%$ of those admitted may require intensive care [12]. Hospitalised COVID-19 patients often have comorbidities, such as cardiovascular disease and diabetes [13] and may have prolonged hospital stays [14]. Some COVID-19 survivors have physical impairments that may be exacerbated by prolonged immobility, including muscle weakness, neurological impairment and/or nutritional disturbance [15]. In-hospital rehabilitation could effectively address these problems [16]. However, there may be added challenges for rehabilitation delivery in the setting of COVID-19, related to infection prevention and changes to availability of existing rehabilitation services.

\section{Results}

The majority of the experts recommended strongly (55\%) or conditionally (37\%) for hospitalised patients with COVID-19 receiving rehabilitation at/around the bedside (critical care and/or ward based) until safe discharge to the home environment (figure la and table 1).

Experts commented that patient-tailored early rehabilitative interventions similar to other critically ill patients should start after an assessment, including early mobilisation and airway clearance. This may prevent and/or slow down the expected rapid deterioration in physical and emotional functioning. Some experts did express concerns about patients' safety, due to the limited understanding of underlying pathophysiology and the possible impact of rehabilitative interventions. For example, it is unclear if there is any "threshold of illness severity" or any particular features of illness, which pose greater risk of patients undertaking rehabilitative interventions during hospitalisation, including pulmonary, cardiovascular and/or neurological manifestations of COVID-19 infection, in particular, the risk of clotting (microthrombosis and venous thromboembolism). Moreover, the importance of appropriate personal protective equipment (PPE) for the healthcare professional was mentioned by multiple experts as a clear safety issue.

The international task force suggests that prior to hospital discharge, hospitalised patients with COVID-19 should have an assessment of oxygen requirements at rest and during exertion Rationale

Hypoxaemia is common in hospitalised patients with COVID-19, with hypoxic respiratory failure a prominent feature of severe cases [17]. While supplemental oxygen requirements reduce over time, alongside improvements in the underlying lung pathology [18], it is likely that some patients will have ongoing oxygen needs at hospital discharge. Moreover, some patients in the recovery phase may have oxygen desaturation on exertion, which is not associated with resting oxygen saturation, the degree of dyspnoea or feeling unwell.

\section{Results}

A majority of the experts recommended strongly (66\%) or conditionally (26\%) for hospitalised patients with COVID-19 having an assessment of oxygen requirements at rest and during exertion prior to hospital discharge (figure 1b).

Experts commented that assessment of oxygen requirements during exertion may vary between hospitals. However, low oxygen saturation may be a reason for additional assessment for pulmonary and cardiovascular comorbidities. Moreover, some patients do show a severe oxygen desaturation during physical exercise of only moderate intensity. Assessment of oxygen requirement prior to hospital discharge is necessary to prepare the individual COVID-19 patient with the needed equipment upon discharge home. A follow-up assessment on the re-evaluation of oxygen requirement should also be scheduled following hospital discharge, as supplemental oxygen may no longer be needed as the underlying lung pathology resolves.

\section{The international task force suggests that patients with COVID-19 should be encouraged to do regular daily activities in the first 6-8 weeks after hospital discharge \\ Rationale}

Some patients with COVID-19 will resume daily activities without difficulty in the period following hospitalisation, but this may be more challenging in those who have had severe disease, prolonged hospital stays and/or persistent symptoms [19]. Approximately two-thirds of the survivors of other critical illnesses experience moderate-to-good recovery of physical function over the first 2 months [20]. However, recovery trajectories vary, with ongoing impairments most likely in older patients with longer intensive care unit (ICU) stays and greater sedation time [20]. 
a) Q1. Should hospitalised patients with COVID-19 receive ehabilitation at/around the bedside Icritical care and/or ward based) until safe for discharge to the hom

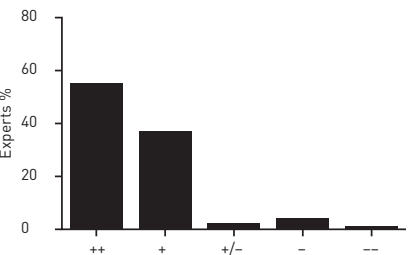

Q4. Should patients with COVID-19 be encouraged to do low-/moderate-intensity physical exercise at home (rather than high intensity physical exercise) in the first assessment with measures of exertionat desaturation assessment with measurs

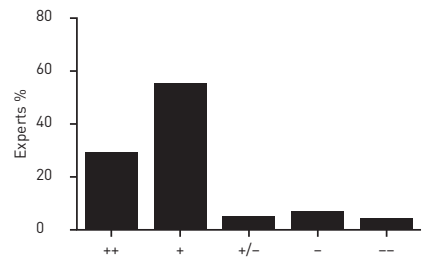

g) Q7. Should follow-up of a hospitalised patient with COVID-19 include measures of respiratory function a 6-8 weeks following hospital discharge?

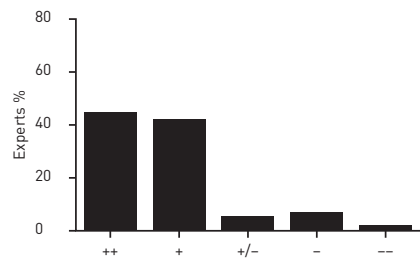

j) Q10. Should COVID-19 survivors with pre-existing/onhospital discharge receive a comprehensive pulmonary rehabilitation programme consistent with established international standards, compared to no pulmonary

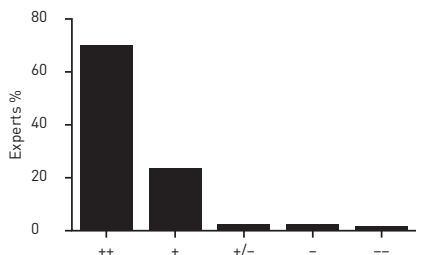

m) Q13. Should COVID-19 survivors with symptoms of psychological distress lassessed using questionnaires mal psychological assessment?

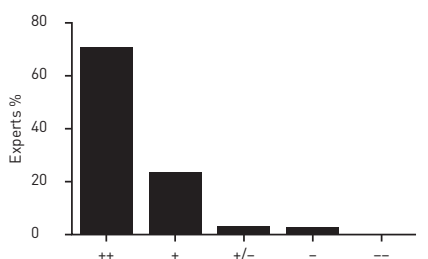
Q) Prior to hospital discharge, should hospitalised patients with covid-19 have an assessm?

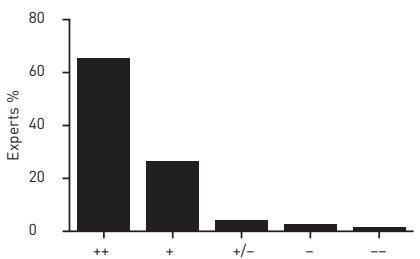

e) Q5. Should with patients with COVID-19 have a forma assessment of physical and emotional functioning a 6-8 weeks following discharge, to identify unmet rehabilitation needs?

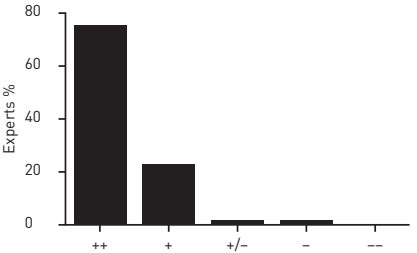

h) Q8. Should follow-up of a hospitalised patient with COVID-19 include measures of exercise capacity at 6-8 weeks following hospital discharge?

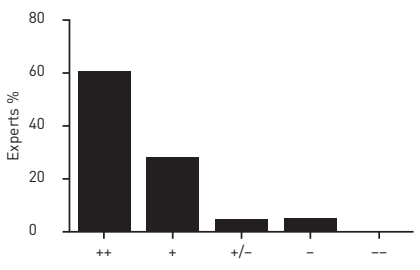

k) Q11. Should COVID-19 survivors with loss of lower-limb muscle mass and/or function at 6-8 weeks following hospital discharge receive a muscle strengthening
programme, rather than no strengthening programme?

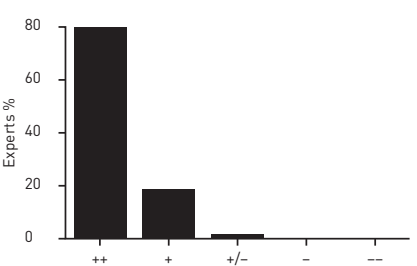

Q3. Should patients with COVID-19 be encouraged to do activities in the first 6-8 weeks after ospital discharge?

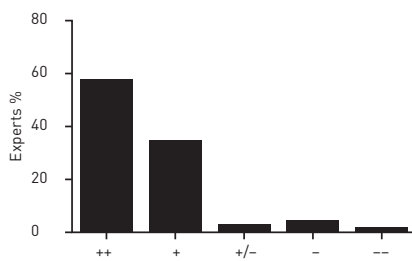

f) Q6. Should follow-up of a hospitalised patient with COVID-19 include the core outcomes set (e.g. EQ-5D, Evospital Anxiety and Depression Scale, Impact of acute respiratory failure at $6-8$ weeks following hospital discharge?

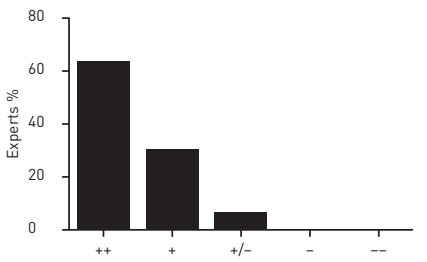

i) Q7. Should COVID-19 survivors with a need for rehabilitative interventions at 6-8 weeks following hospital discharge le.g. multiple treatable traits comper a comprehensive rehabilitation programm

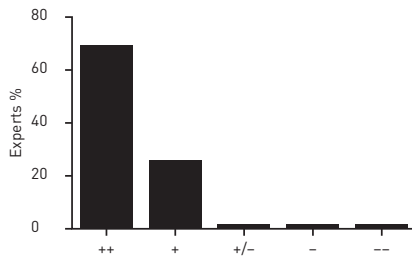

I) Q12. Should COVID-19 survivors with loss of lower-limb muscle mass at 6-8 weeks following hospital discharge receive nutritional support rather than no nutritiona support?

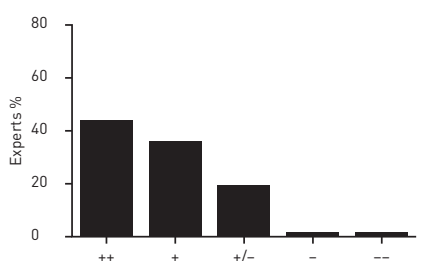

FIGURE 1 Experts' responses to the 13 questions. COVID-19: coronavirus disease 2019; ++: strong recommendation for; +: conditional recommendation for; +/-: no recommendation for or against; -: conditional recommendation against; --: strong recommendation against.

Results

A majority of the experts recommended strongly (58\%) or conditionally (34\%) for encouraging patients with COVID-19 to do regular daily activities in the first 6-8 weeks after hospital discharge (figure 1c).

Experts commented that recommencing daily physical activity as early as possible may positively affect functional recovery. However, patients may vary in physical, emotional and/or cognitive functioning, so 
TABLE 1 Interim recommendations

1. Hospitalised patients with COVID-19 should receive rehabilitation at/around the bedside /critical care and/or ward based), until safe for discharge to the home environment.

2. Prior to hospital discharge, hospitalised patients with COVID-19 should have an assessment of oxygen requirements at rest and during exertion.

3. Patients with COVID-19 should be encouraged to do regular daily activities in the first 6-8 weeks after hospital discharge.

4. Patients with COVID-19 should be encouraged to do low-/moderate-intensity physical exercise at home (rather than high-intensity physical exercise) in the first 6-8 weeks after hospital discharge, if a formal exercise assessment with measures of exertional desaturation has not been conducted.

5. Patients with COVID-19 should have a formal assessment of physical and emotional functioning at 6-8 weeks following discharge, to identify unmet rehabilitation needs.

6. Follow-up of a hospitalised patient with COVID-19 should include the core outcomes set for survivors of acute respiratory failure at 6-8 weeks following hospital discharge.

7. Follow-up of a hospitalised patient with COVID-19 should include measures of respiratory function at 6-8 weeks following hospital discharge.

8. Follow-up of a hospitalised patient with COVID-19 should include measures of exercise capacity at 6-8 weeks following hospital discharge.

9. COVID-19 survivors with a need for rehabilitative interventions at 6-8 weeks following hospital discharge (e.g. multiple treatable traits) should receive a comprehensive rehabilitation programme, compared to no rehabilitation programme.

10. COVID-19 survivors with pre-existing/ongoing lung function impairment at 6-8 weeks following hospital discharge should receive a comprehensive pulmonary rehabilitation programme consistent with established international standards, compared to no pulmonary rehabilitation programme.

11. COVID-19 survivors with loss of lower-limb muscle mass and/or function at 6-8 weeks following hospital discharge should receive a muscle strengthening programme, rather than no strengthening programme.

12. COVID-19 survivors with loss of lower-limb muscle mass at 6-8 weeks following hospital discharge should receive nutritional support rather than no nutritional support.

13. COVID-19 survivors with symptoms of psychological distress (assessed using questionnaires) at 68 weeks after discharge from the hospital should receive a formal psychological assessment.

COVID-19: coronavirus disease 2019

further assessment should be performed. Moreover, the encouragement to do regular daily activities should be in line with the local regulations for physical distancing and should go along with advice to take time for recovery and rest periods, and taking an acceptable level of perceived exertion and dyspnoea and oxygen desaturation into consideration.

The international task force suggests that patients with COVID-19 should be encouraged to do low-/moderate-intensity physical exercise at home (rather than high-intensity physical exercise) in the first 6-8 weeks after hospital discharge, if a formal exercise assessment with measures of exertional desaturation has not been conducted

Rationale

COVID-19 infection is associated with a high inflammatory burden [21], which could persist beyond hospital discharge. Exertional desaturation may also persist, even in those without the requirement for supplemental oxygen at rest. While moderate-intensity exercise training is safe and feasible in survivors of critical illness [22], the safety of high-intensity exercise in patients recovering from COVID-19 is unknown. During the early post-hospital period it may not always be possible to conduct a robust exercise assessment or deliver supervised rehabilitation services, with uncertainties regarding the duration of time that patients remain infectious [23] and/or the need to maintain physical distancing.

Results

A majority of the experts recommended strongly (29\%) or conditionally (55\%) for encouraging patients with COVID-19 to do low-/moderate-intensity physical exercise at home (rather than high-intensity physical exercise) in the first 6-8 weeks after hospital discharge, if a formal exercise assessment with measures of exertional desaturation has not been conducted (figure 1d).

Some experts stated that the potential benefits of low-/moderate-physical activity in preventing physical deterioration outweighed any perceived risks of undertaking such exercise without formal assessment. The low-/moderate-intensity physical exercises should be based on symptom limitation and tailored to the 
individual needs and limitations of the patients, which may vary greatly. In addition to oxygen desaturation on exertion, experts encouraged health professionals to be aware of other possible causes of caution, such as cardiomyopathy and (post-)pulmonary embolism.

The international task force suggests that patients with COVID-19 should have a formal assessment of physical and emotional functioning at 6-8 weeks following discharge, to identify unmet rehabilitation needs

Rationale

While good recovery of physical function should be expected over the first 8 weeks in most COVID-19 survivors, it is highly likely that there will be some with ongoing impairments in physical functioning [20]. The high prevalence of post-traumatic stress disorder (PTSD), cognitive impairment, chronic pain, sleep disorders, fibromyalgia and fatigue in survivors of critical illness have been well documented [24-26]. The COVID-19 pandemic gives rise to new psychosocial and emotional stressors for recovering patients, including social isolation, physical distancing, loss of employment and uncertainties about the future.

\section{Results}

A majority of the experts recommended strongly (75\%) or conditionally (22\%) for patients with COVID-19 having a formal assessment of physical and emotional functioning at 6-8 weeks following discharge (figure 1e).

Experts stated that post-hospitalisation recovery may vary considerably between patients, which justifies a formal assessment (including physical, emotional and cognitive functioning and return to work) to customise rehabilitation care. In addition, some experts proposed an evaluation at hospital discharge to ensure that patients were discharged to the appropriate setting (e.g. home, rehabilitation centre, nursing home), and for formal assessment only in symptomatic patients with limitations in daily functioning.

The international task force suggests that follow-up of a hospitalised patient with COVID-19 should include the core outcomes set for survivors of acute respiratory failure at 6-8 weeks following hospital discharge

Rationale

Post-hospital outcomes of COVID-19 survivors are not yet understood. Assessment of physical and psychosocial outcomes following hospitalisation for COVID-19 will allow unmet rehabilitation needs to be addressed. A core outcome set allows essential outcomes to be consistently assessed using the same measurement instruments, so that data can be pooled and confident conclusions drawn. A core outcome set for survivors of acute respiratory failure has already been defined using a consensus process, and includes the EQ-5D, Hospital Anxiety and Depression Scale and Impact of Events Scale - Revised [27]. The core outcome set does not include measures of cognition, muscle function, physical function or pulmonary function.

Results

A majority of the experts recommended strongly (63\%) or conditionally (30\%) for the follow-up of a hospitalised patient with COVID-19 including the core outcomes set for survivors of acute respiratory failure at 6-8 weeks following hospital discharge (figure 1f)

Experts recognised the importance of administering outcome measures that will help to identify the patients' need in order to direct, plan and devise appropriate interventions and resources to the patients and their caregivers. This may require multiple re-assessments at different time points (e.g. 3, 6 and 12 months after hospital discharge). Individual experts suggested the addition of a tool to measure exertional breathlessness (e.g. the Medical Research Council dyspnoea grading scale) and cognitive screening (Montreal Cognitive Assessment) and alternative tools to assess the same domains as identified by NeEDHam et al. [27] (e.g. Short-Form 36 to assess generic health status; the primary care PTSD questionnaire to assess symptoms of PTSD; Patient Health Questionnaire 9, General Anxiety Disorder 7 or the Depression Anxiety Stress Scale 21 to assess symptoms of anxiety and/or depression). Some experts believed that assessment at 6-8 weeks following hospital discharge is too soon, as some sequelae may manifest at later time points. Moreover, some experts indicted that community-managed patients may also need a supported recovery programme.

The international task force suggests that follow-up of a hospitalised patient with COVID-19 should include measures of respiratory function at 6-8 weeks following hospital discharge Rationale

High-resolution computed tomography scans of the chest in patients with COVID-19 infection have shown significant respiratory abnormalities including ground-glass opacities, inter- and intra-lobular septal 
thickening and consolidation. These changes start to resolve after 14 days, but may persist past hospital discharge [18]. Long-term follow-up of survivors of other severe viral pneumonias (SARS-CoV-1 and Middle East respiratory syndrome (MERS)-CoV) suggests that many had normal respiratory function at 1 year, although impaired diffusing capacity was evident in a minority [28, 29]. Respiratory function testing is necessary to document ongoing impairments and guide future management, but it is considered an aerosol-generating procedure, which may limit its availability in the COVID-19 era.

\section{Results}

A majority of the experts recommended strongly (45\%) or conditionally (42\%) for the follow-up of a hospitalised patient with COVID-19 including measures of respiratory function at 6-8 weeks following hospital discharge (figure $1 \mathrm{~g}$ ).

Experts recognised the importance of measuring respiratory function, as missing respiratory abnormalities are likely to lead to further decline, and perhaps downstream, new respiratory chronic disease. Experts emphasised the need to perform assessment only in patients with lingering respiratory symptoms and who were tested COVID-negative in the post-hospitalisation phase. Indeed, standard operating procedures need to be in place to prevent possible infection of other patients and/or the lung-function technician. Some experts questioned whether local infection control protocols would allow measurements of respiratory function to be obtained.

The international task force suggests that follow-up of a hospitalised patient with COVID-19 should include measures of exercise capacity at 6-8 weeks following hospital discharge Rationale

While the long-term consequences of COVID-19 are not fully understood, we know that the impact of an ICU stay for acute respiratory distress syndrome (ARDS) has a significant impact on physical function [30]. Equally, a protracted period of bed rest can have similar detrimental but partially reversible effects in chronic respiratory disease [31]. There is some evidence that recovery in physical function is variable in ARDS survivors; however, the greatest recovery appeared to occur within the first 2 months [20]. An objective measure of exercise capacity [31] is important to document the degree of disability, identify the limitations to exercise and form the foundation of a subsequent rehabilitation programme.

Results

A majority of the experts recommended strongly (61\%) or conditionally (29\%) for the follow-up of a hospitalised patient with COVID-19 including measures of exercise capacity at 6-8 weeks following hospital discharge (figure $1 \mathrm{~h}$ ).

Many experts considered the measurement of exercise capacity to be very important in COVID-19 survivors, but some experts believe that this measurement should be limited to patients who remain physically limited 6-8 weeks following hospital discharge and who are tested negative at the time testing the exercise capacity. Experts emphasised that assessors should be aware of the possible presence of cardiopulmonary sequelae. Some experts questioned whether local infection control protocols would allow measurements of exercise capacity to be obtained.

The international task force suggests that COVID-19 survivors with a need for rehabilitative interventions at 6-8 weeks following hospital discharge (e.g. multiple treatable traits) should receive a comprehensive rehabilitation programme, compared to no rehabilitation programme Rationale

Existing data from survivors of viral pneumonias indicates the wide range of challenges that patients face $[20,24,25]$. It is unlikely that a unidimensional programme of physical training will meet the needs of COVID-19 survivors as they will exhibit multiple treatable traits that a comprehensive rehabilitation programme has the potential to modify favourably. There is a limited evidence base for pulmonary rehabilitation post-H1N1-ARDS [32]. The programme will potentially be wider in scope than current pulmonary rehabilitation programmes [33] to meet the needs of these individuals and consider the additional burden placed upon survivors as a consequence of this unique virus, e.g. social isolation strategies and the associated emotional burden. Survivors may be of a different age group to the "usual" pulmonary rehabilitation population and supporting a successful return to work will be important.

Results

A majority of the experts recommended strongly (70\%) or conditionally (26\%) for COVID-19 survivors with a need for rehabilitative interventions at 6-8 weeks following hospital discharge receiving a comprehensive rehabilitation programme (figure 1i). 
Many experts commented on the diverse needs of COVID-19 survivors, such that not all survivors will need a comprehensive programme. Some experts commented that a comprehensive rehabilitation programme may not be available in all locations during the COVID-19 pandemic.

The international task force suggests that COVID-19 survivors with pre-existing/ongoing lung function impairment at 6-8 weeks following hospital discharge should receive a comprehensive pulmonary rehabilitation programme consistent with established international standards, compared to no pulmonary rehabilitation programme Rationale

For patients with COPD there is evidence to indicate that recovery after an admission is supported by a structured rehabilitation programme. We know that this is both safe and clinically effective and is recommended in national and international guidelines [33, 34]. The current guidance for routine post-exacerbation pulmonary rehabilitation is 4 weeks post-discharge; however, the recommendation to delay this until 6-8 weeks post-discharge was based upon the following considerations: there is a lack of data about the decay of the levels of infection in the COVID-19 survivor; the data suggest that at 2 months a proportion of physical recovery will have occurred [20]; and we know that is a challenge for services to recruit patients to a post-exacerbation rehabilitation programme 4 weeks post-discharge [35].

Results

A majority of the experts recommended strongly (70\%) or conditionally (24\%) for COVID-19 survivors with pre-existing/ongoing lung function impairment at 6-8 weeks following hospital discharge receiving a comprehensive pulmonary rehabilitation programme consistent with established international standards (figure $1 \mathrm{j}$ ).

Experts recommended taking patient's individual needs and preferences into consideration when decisions regarding comprehensive pulmonary rehabilitation programmes are made.

The international task force suggests that COVID-19 survivors with loss of lower-limb muscle mass and/or function at 6-8 weeks following hospital discharge should receive a muscle-strengthening programme, rather than no strengthening programme Rationale

For patients with ARDS, prolonged stay in the ICU (mostly including prolonged mechanical ventilation) is known to have significant impact on peripheral muscle function, reflected in a loss of muscle mass and power neuropathy and/or myopathy, better known as ICU-acquired muscle weakness [30], and occurs early on in the ICU stay [36]. Equally, a prolonged period of bed rest can also have a significant effect in chronic respiratory disease [31]. The lack of peripheral muscle strength can severely compromise functional ability and should be evaluated and treated as indicated [33].

\section{Results}

A majority of the experts recommended strongly (80\%) or conditionally (18\%) for COVID-19 survivors with loss of lower limb muscle mass and/or function at 6-8 weeks following hospital discharge receiving a muscle strengthening programme (figure $1 \mathrm{k}$ ).

Experts agreed that a muscle strengthening programme was important to optimise recovery. Moreover, muscle strength needs to be assessed prior to commencement, to enable accurate prescription and tailoring of the strengthening programme.

The international task force suggests that COVID-19 survivors with loss of lower-limb muscle mass at 6-8 weeks following hospital discharge should receive nutritional support rather than no nutritional support

Rationale

It is well established that optimising caloric and protein intake is important to support recovery of functional muscle mass. The post-ICU COVID-19 survivor will face commonly reported problems with nutrition (e.g. loss of appetite, swallowing disorders), and the additional symptoms of loss of taste (ageusia) and smell (anosmia) are now recognised symptoms of COVID-19 [37]. In general, for those with chronic respiratory disease, weight loss and wasting of muscle and bone tissue may be induced or accelerated during severe acute exacerbations of respiratory disease requiring hospitalisation, due to the combination of malnutrition, physical inactivity, hypoxia, systemic inflammation and/or systemic glucocorticoids [38]; this is likely to be mirrored in the COVID patient. 
Results

A majority of the experts recommended strongly (43\%) or conditionally (36\%) for COVID-19 survivors with loss of lower-limb muscle mass at 6-8 weeks following hospital discharge receiving nutritional support (figure 11).

Experts stated that rebuilding muscle mass requires that nutritional support be combined with a training stimulus. Moreover, experts believed that nutritional support may be less well established in some centres and guidance may be needed from expert sources.

\section{The international task force suggests that COVID-19 survivors with symptoms of psychological distress lassessed using questionnaires) at 6-8 weeks after discharge from the hospital should receive a formal psychological assessment \\ Rationale}

Common symptoms reported 1 year later by ICU survivors, including patient with ARDS, include anxiety (34\%), depression (33\%) and PTSD (19\%) [39]. This is compounded for the post-COVID-19 survivor by the emotional stress associated with social isolation from family and friends for a protracted period of time as a consequence of government lockdown policies. There are questionnaires recommended in the core outcome dataset that cover these aspects of anxiety and depression, alongside PTSD [27]. In addition, it is acknowledged that the emotional burden of COVID-19 is likely to extend beyond the individual to family and friends [40].

\section{Results}

A majority of the experts recommended strongly (71\%) or conditionally (24\%) for COVID-19 survivors with symptoms of psychological distress at 6-8 weeks after discharge from the hospital receiving a formal psychological assessment (figure $1 \mathrm{~m}$ ).

Experts believed that symptoms of anxiety, depression and PTSD occur commonly in persons after a major life event. Therefore, screening and monitoring of the course of symptoms of psychological distress is important. Experts also emphasised that those patients who show high levels of anxiety or depressive symptoms need to be referred to psychologist or psychiatrist for further assessment, and treatment may be indicated when symptoms continue to exist after 10-12 weeks. Psychological assessment and support for the family of the infected patient was also emphasised by the experts.

\section{Discussion}

The present study provides consensus-based suggestions for the screening and the rehabilitation process during and after a hospital admission for severe COVID-19 infection. Experts reached consensus based upon indirect evidence and nonsystematic clinical observations (i.e. clinical experience) on the need for early rehabilitation during the hospital admission, the screening for treatable traits with rehabilitation in all patients at discharge and 6-8 weeks after discharge, and around the content of rehabilitation for these patients. In absence of a formal evidence-based approach, these findings provide interim guidance for referral and multidisciplinary rehabilitation in a subgroup of patients after hospital admission. Altogether, the data cast a strong claim on the need for screening and rehabilitation options for patients who have been hospitalised. Besides lung function testing (e.g. spirometry, whole-body plethysmography and carbon monoxide transfer factor) [41, 42], this screening should also contain at least an exercise test (cardiopulmonary exercise test, 6-min walk test or shuttle walk test), muscle strength testing and patient-reported outcome measures [19, 43-46]. This cannot be ignored by healthcare systems organising the care around this pandemic respiratory infection. It should be noted that several experts identified that during the pandemic there has been an absence of rehabilitation options for patients who suffered from COVID-19.

Early mobilisation and re-engagement in physical activity is important in the prevention of systemic consequences of a critical care and hospital admission. Whenever possible, patients should re-engage in physical exercise tailored to their possibilities. In patients suffering from COVID-19, such early interventions have to be balanced with the critical illness of the patient and the availability of PPE for caregivers engaged in the early mobilisation protocols $[5,6,47]$. Where rehabilitation staff are not available or cannot access PPE, some rehabilitation tasks at the bedside may be provided by other members of the multidisciplinary team, with appropriate instruction. At discharge from hospital, experts advise a first screening, particularly to investigate the need for oxygen supplementation, in order to guide the first weeks post-discharge. Patients may still be infectious [48, 49], which may complicate testing outside the patient's room. A 1-min sit-to-stand test has been proposed as a way to evaluate hypoxia on exertion in the patient's room as an alternative to a 6-min walk test [50], but needs further validation. 
After discharge, patients with COVID-19 should be encouraged to do low-/moderate-intensity physical exercise at home in the first 6-8 weeks. Usually, these tasks range between 1.5 and 6 metabolic equivalents; AINSWORTH and co-workers [51, 52] describe numerous examples.

Moreover, the guidance provided by the ATS/ERS statement on pulmonary rehabilitation [33] may serve as a good framework. In this statement, pulmonary rehabilitation is defined as "...a comprehensive intervention based on a thorough patient assessment followed by patient-tailored therapies, which include, but are not limited to, exercise training, education, and behaviour change, designed to improve the physical and psychological condition of people with chronic respiratory disease and to promote the long-term adherence of health-enhancing behaviours". Experts seem to agree that this definition also applies to patients who have suffered severe COVID-19. Clearly there is a need for physiological (statements 2, 5, 7, 8 and 11), psychological (statements 5, 6 and 13) and nutritional (statement 13) assessment. For some patients there is a need for an individually tailored rehabilitation intervention (statement 9) and in the case of a pre-existing of remaining respiratory deficit, a pulmonary rehabilitation programme (statement 10). Pulmonary rehabilitation programmes are indeed well equipped to service this group of patients. Adaptations may need to be made to the rehabilitation programme if the programme typically deals with patients with obstructive lung disease, to cater more to patients with gas-exchange abnormalities. The self-management or education modules may also need to be adapted. It is expected that many patients who have suffered from severe COVID-19, and particularly those with an ICU admission, will have significant skeletal muscle weakness. Following muscle strength testing [46], specific skeletal muscle training programmes, typically offered with resistance training, are advised by experts. This is also the case in conventional pulmonary rehabilitation [53].

To date, no prospective studies exist in COVID-19 patients on the proportion of patients in need of some form of (multidisciplinary) rehabilitation. However, symptoms like fatigue and dyspnoea still occur frequently 30 days after discharge from the hospital, and are accompanied by reduced quality of life in $\sim 40 \%$ of COVID-19 patients [19]. In some cases, the programme offered could be outside the pulmonary rehabilitation setting. Indeed, early studies from China and confirmed later [54] indicated that $>30 \%$ of patients suffered from some neurological and neuromuscular sequelae [55]. It is yet unknown how much of this recovers spontaneously. The progression of functional, skeletal muscle and mental health status after an admission for COVID-19 is not yet well described. Nevertheless, observational studies after SARS [56] or MERS [57] report an impaired 6-min walk distance in $18 \%$ of the patients, and $43.6 \%$ had psychological comorbidity. Hence, healthcare systems need to prepare to accommodate an increased number of referrals.

The exact proportion of COVID-19 patients requiring rehabilitation is difficult to predict, but assuming that patients who received mechanical ventilation would be a minimum set of patients that require rehabilitation post-discharge may provide a conservative estimate of the number of new rehabilitation cases. Taking the International Severe Acute Respiratory and Emerging Infection Consortium (ISARIC), UK-based database as an example, $10 \%$ of hospitalised patients required mechanical ventilation [58]. In a United States (New York)-based cohort, 23\% of patients required mechanical ventilation [59]. Unfortunately, large proportions died in the ICU (e.g. up to $60 \%$ in a study from New York, and similar numbers in the ISARIC cohort), still rendering $40 \%$ of patients admitted to ICU as minimal numbers of new candidates for multidisciplinary rehabilitation, representing $5-10 \%$ of patients referred to hospital. It is likely that a fraction of patients not mechanically ventilated (including patients on high-flow oxygen therapy) will need to be added, as some of these still have significant functional consequences of the hospital admission ([60]; M Paneroni, Respiratory Rehabilitation of the Institute of Lumezzane, Instituti Clinici Scientifici Maugeri IRCCS, Lumezzane, Italy; personal communication). We strongly encourage countries to take action to organise rehabilitation for this influx of patients, in addition to the many patients with chronic respiratory disease who already qualify for pulmonary rehabilitation [61].

An issue that is unresolved, but of importance to the organisation of rehabilitation for these patients, is the duration for which patients should be considered infectious. There is currently no consensus on how long patients should be self-isolating. Local infection prevention recommendations should be followed and this may require significant adaptation of the rehabilitation programme with, for example, the adoption of "tele-rehabilitation" [62]. The task force has adopted a 6-8-week time window for reassessment. At this time point (and probably even sooner) many patients will be considered noninfectious $[48,49,63]$.

A last point of attention is that despite the robust opinion in favour of rehabilitation after severe COVID-19 disease, these patients may be difficult to reach. Unfortunately, several factors may preclude referral or uptake of rehabilitation. An important element that may impair uptake of rehabilitation is that at a population level, rehabilitation might be hampered by pre-infection risk factors for admission to hospital with COVID-19, which include obesity, smoking and living an inactive lifestyle [64]. Moreover, it 
is known that COVID-19 disproportionately affects disadvantaged communities [65], many of whom have poor access to rehabilitation [66]. These factors are known to impair uptake of rehabilitation. Therefore, the offer of rehabilitation must be made to patients in a personalised and targeted manner, to maximise the likelihood of acceptance.

\section{Strengths and weaknesses}

While the present expert opinion article followed rigorous methodology to reach consensus, and consensus was reached on all questions, some remarks need to be taken into account. It would be good to offer respondents the option of no response, as respondents may feel that they do not have adequate expertise to respond to a question or they may not understand the question wording. Indeed, some experts stated that there were composite questions, combining a question about the content of care with a question about timing. Moreover, sometimes questions may not have been very specific (e.g. "measures of respiratory function", "measures of exercise capacity", "comprehensive pulmonary rehabilitation programme" or "nutritional support"). See supplementary table S1 for all details. This may complicate interpretation of survey responses. Where possible, we have taken written comments of the respondents into account.

The current methodology also has strengths. It is fast and provides an interim guidance when randomised controlled studies are not yet available. Moreover, the current sample experts came from all over the world and had a diverse professional background, including but not limited to medical specialists, physiotherapists, nurses and psychologists.

\section{Conclusion}

This multinational task force recommends early bedside rehabilitation for patients affected by severe COVID-19. It advocates for assessment of oxygen needs at discharge and more comprehensive assessment of rehabilitation needs including physical as well as mental aspects 6-8 weeks after discharge. Based on the deficits identified multidisciplinary rehabilitation should be offered with attention for skeletal muscle and functional as well as mental restoration. The model of pulmonary rehabilitation may suit as a framework, particularly in a subset of patients with pre-existing or COVID-19-induced long-term respiratory consequences.

Acknowledgements: The authors would like to thank Alessandra Marguerat (European Respiratory Society, Lausanne, Switzerland) for her logistical support. Moreover, they would like to acknowledge the experts (supplementary table S2) for their time and input.

Conflict of interest: M.A. Spruit reports grants from Netherlands Lung Foundation and Stichting Astma Bestrijding, grants and personal fees from AstraZeneca and Boehringer Ingeheim, outside the submitted work. A.E. Holland has nothing to disclose. S.J. Singh has nothing to disclose. T. Tonia acts as an ERS methodologist. K.C. Wilson reports other possible conflicts of interest as ATS Chief of Guidelines and Documents, and as developer of the CORE process, outside the submitted work. T. Troosters has nothing to disclose.

\section{References}

1 Johns Hopkins University \& Medicine Coronavirus Resource Center. COVID-19 Dashboard. https://coronavirus. jhu.edu/map.html Date last updated: August 18, 2020. Date last accessed: July 14, 2020.

2 Docherty AB, Harrison EM, Green CA, et al. Features of 20133 UK patients in hospital with covid-19 using the ISARIC WHO Clinical Characterisation Protocol: prospective observational cohort study. BMJ 2020; 369: m1985.

3 Polastri M, Nava S, Clini E, et al. COVID-19 and pulmonary rehabilitation: preparing for phase three. Eur Respir J 2020; 55: 2001822

4 Vitacca M, Lazzeri M, Guffanti E, et al. Italian suggestions for pulmonary rehabilitation in COVID-19 patients recovering from acute respiratory failure: results of a Delphi process. Monaldi Arch Chest Dis 2020; 90: 10.4081/ monaldi.2020.1444.

5 Vitacca M, Carone M, Clini EM, et al. Joint statement on the role of respiratory rehabilitation in the COVID-19 crisis: the Italian position paper. Respiration 2020; 99: 493-499.

6 Thomas P, Baldwin C, Bissett B, et al. Physiotherapy management for COVID-19 in the acute hospital setting: clinical practice recommendations. J Physiother 2020; 66: 73-82.

7 Pan American Health Organization. Rehabilitation Considerations During the COVID-19 Outbreak. https://iris. paho.org/bitstream/handle/10665.2/52035/NMHMHCOVID19200010_eng.pdf?sequence=6\&isAllowed=y

8 Wilson KC, Cotirmall SJ, Bai C, et al. COVID-19: Interim Guidance on Management Pending Empirical Evidence. From an American Thoracic Society-led International Task Force. www.thoracic.org/covid/covid-19-guidance.pdf

9 Wilson KC, Schoenberg NC, Raghu G. Idiopathic pulmonary fibrosis guideline recommendations. Need for adherence to Institute of Medicine methodology? Ann Am Thorac Soc 2019; 16: 681-686.

10 Spruit M. COVID-19 and Rehabilitation. www.ersnet.org/covid-19-blog/covid-19-and-rehabilitation Date last updated: April 3, 2020.

11 Gandhi RT, Lynch JB, Del Rio C. Mild or moderate covid-19. N Engl J Med 2020; 383: 1757-1766.

12 Rodriguez-Morales AJ, Cardona-Ospina JA, Gutiérrez-Ocampo E, et al. Clinical, laboratory and imaging features of COVID-19: a systematic review and meta-analysis. Travel Med Infect Dis 2020; 34: 101623.

13 Emami A, Javanmardi F, Pirbonyeh N, et al. Prevalence of underlying diseases in hospitalized patients with COVID-19: a systematic review and meta-analysis. Arch Acad Emerg Med 2020; 8: e35. 
14 Wang L, He W, Yu X, et al. Coronavirus disease 2019 in elderly patients: characteristics and prognostic factors based on 4-week follow-up. J Infect 2020; 80: 639-645.

15 Kiekens C, Boldrini P, Andreoli A, et al. Rehabilitation and respiratory management in the acute and early post-acute phase. "Instant paper from the field" on rehabilitation answers to the COVID-19 emergency. Eur J Phys Rehabil Med 2020; 56: 323-326.

16 Tipping CJ, Harrold M, Holland A, et al. The effects of active mobilisation and rehabilitation in ICU on mortality and function: a systematic review. Intensive Care Med 2017; 43: 171-183.

17 Bhatraju PK, Ghassemieh BJ, Nichols M, et al. Covid-19 in critically ill patients in the Seattle region - case series. N Engl J Med 2020; 382: 2012-2022.

18 Pan F, Ye T, Sun P, et al. Time course of lung changes at chest CT during recovery from coronavirus disease 2019 (COVID-19). Radiology 2020; 295: 715-721.

19 Carfi A, Bernabei R, Landi F, et al. Persistent symptoms in patients after acute COVID-19. JAMA 2020; 324: 603-605.

20 Gandotra S, Lovato J, Case D, et al. Physical function trajectories in survivors of acute respiratory failure. Ann Am Thorac Soc 2019; 16: 471-477.

21 Madjid M, Safavi-Naeini P, Solomon SD, et al. Potential effects of coronaviruses on the cardiovascular system: a review. JAMA Cardiol 2020; 5: 831-840.

22 Denehy L, Skinner EH, Edbrooke L, et al. Exercise rehabilitation for patients with critical illness: a randomized controlled trial with 12 months of follow-up. Crit Care 2013; 17: R156.

23 Liu Y, Yan LM, Wan L, et al. Viral dynamics in mild and severe cases of COVID-19. Lancet Infect Dis 2020; 20: 656-657.

24 Pandharipande PP, Girard TD, Jackson JC, et al. Long-term cognitive impairment after critical illness. $N$ Engl J Med 2013; 369: 1306-1316.

25 Parker AM, Sricharoenchai T, Raparla S, et al. Posttraumatic stress disorder in critical illness survivors: a metaanalysis. Crit Care Med 2015; 43: 1121-1129.

26 Vittori A, Lerman J, Cascella M, et al. COVID-19 pandemic acute respiratory distress syndrome survivors: pain after the storm? Anesth Analg 2020; 131: 117-119.

27 Needham DM, Sepulveda KA, Dinglas VD, et al. Core outcome measures for clinical research in acute respiratory failure survivors. An international modified Delphi consensus study. Am J Respir Crit Care Med 2017; 196: $1122-1130$.

28 Tansey CM, Louie M, Loeb M, et al. One-year outcomes and health care utilization in survivors of severe acute respiratory syndrome. Arch Intern Med 2007; 167: 1312-1320.

29 Park WB, Jun KI, Kim G, et al. Correlation between pneumonia severity and pulmonary complications in Middle East respiratory syndrome. J Korean Med Sci 2018; 33: e169.

30 Herridge MS, Tansey CM, Matté A, et al. Functional disability 5 years after acute respiratory distress syndrome. N Engl J Med 2011; 364: 1293-1304

31 Spruit MA, Gosselink R, Troosters T, et al. Muscle force during an acute exacerbation in hospitalised patients with COPD and its relationship with CXCL8 and IGF-I. Thorax 2003; 58: 752-756.

32 Hsieh MJ, Lee WC, Cho HY, et al. Recovery of pulmonary functions, exercise capacity, and quality of life after pulmonary rehabilitation in survivors of ARDS due to severe influenza A (H1N1) pneumonitis. Influenza Other Respir Viruses 2018; 12: 643-648.

33 Spruit MA, Singh SJ, Garvey C, et al. An official American Thoracic Society/European Respiratory Society statement: key concepts and advances in pulmonary rehabilitation. Am J Respir Crit Care Med 2013; 188: e13-e64.

34 Bolton CE, Bevan-Smith EF, Blakey JD, et al. British Thoracic Society guideline on pulmonary rehabilitation in adults. Thorax 2013; 68: Suppl. 2, ii1-ii30.

35 Jones SE, Green SA, Clark AL, et al. Pulmonary rehabilitation following hospitalisation for acute exacerbation of COPD: referrals, uptake and adherence. Thorax 2014; 69: 181-182.

36 Connolly B, O’Neill B, Salisbury L, et al. Physical rehabilitation interventions for adult patients during critical illness: an overview of systematic reviews. Thorax 2016; 71: 881-890.

37 Liu JY, Chen TJ, Hwang SJ. Analysis of imported cases of COVID-19 in Taiwan: a nationwide study. Int J Environ Res Public Health 2020; 17: 3311.

38 Schols AM, Ferreira IM, Franssen FM, et al. Nutritional assessment and therapy in COPD: a European Respiratory Society statement. Eur Respir J 2014; 44: 1504-1520.

39 Dijkstra-Kersten SMA, Kok L, Kerckhoffs MC, et al. Neuropsychiatric outcome in subgroups of Intensive Care Unit survivors: Implications for after-care. J Crit Care 2020; 55: 171-176.

40 Cairns PL, Buck HG, Kip KE, et al. Stress management intervention to prevent post-intensive care syndrome-family in patients' spouses. Am J Crit Care 2019; 28: 471-476.

41 Mo X, Jian W, Su Z, et al. Abnormal pulmonary function in COVID-19 patients at time of hospital discharge. Eur Respir J 2020; 55: 2001217.

42 Frija-Masson J, Debray M-P, Gilbert M, et al. Functional characteristics of patients with SARS-CoV-2 pneumonia at 30 days post-infection. Eur Respir J 2020; 56: 2001754.

43 Radtke T, Crook S, Kaltsakas G, et al. ERS statement on standardisation of cardiopulmonary exercise testing in chronic lung diseases. Eur Respir Rev 2019; 28: 180101.

44 Holland AE, Spruit MA, Troosters T, et al. An official European Respiratory Society/American Thoracic Society technical standard: field walking tests in chronic respiratory disease. Eur Respir J 2014; 44: 1428-1446.

45 Singh SJ, Puhan MA, Andrianopoulos V, et al. An official systematic review of the European Respiratory Society/ American Thoracic Society: measurement properties of field walking tests in chronic respiratory disease. Eur Respir J 2014; 44: 1447-1478.

46 Maltais F, Decramer M, Casaburi R, et al. An official American Thoracic Society/European Respiratory Society statement: update on limb muscle dysfunction in chronic obstructive pulmonary disease. Am J Respir Crit Care Med 2014; 189: e15-e62.

47 Lazzeri M, Lanza A, Bellini R, et al. Respiratory physiotherapy in patients with COVID-19 infection in acute setting: a Position Paper of the Italian Association of Respiratory Physiotherapists (ARIR). Monaldi Arch Chest Dis 2020; 90: doi:10.4081/monaldi.2020.1285. 
48 Fu Y, Han P, Zhu R, et al. Risk factors for viral RNA shedding in COVID-19 patients. Eur Respir J 2020; 56 : 2001190.

49 Yan D, Liu XY, Zhu YN, et al. Factors associated with prolonged viral shedding and impact of lopinavir/ritonavir treatment in hospitalised non-critically ill patients with SARS-CoV-2 infection. Eur Respir J 2020; 56: 2000799.

50 Simonelli C, Paneroni M, Fokom AG, et al. How the COVID-19 infection tsunami revolutionized the work of respiratory physiotherapists: an experience from Northern Italy. Monaldi Arch Chest Dis 2020; 90: doi:10.4081/ monaldi.2020.1085.

51 Ainsworth BE, Haskell WL, Herrmann SD, et al. 2011 compendium of physical activities: a second update of codes and MET values. Med Sci Sports Exerc 2011; 43: 1575-1581.

52 Ainsworth BE, Haskell WL, Whitt MC, et al. Compendium of physical activities: an update of activity codes and MET intensities. Med Sci Sports Exerc 2000; 32: Suppl. 9, S498-S504.

53 Spruit MA, Wouters EFM. Organizational aspects of pulmonary rehabilitation in chronic respiratory diseases. Respirology 2019; 24: 838-843.

54 Mao L, Jin H, Wang M, et al. Neurologic manifestations of hospitalized patients with coronavirus disease 2019 in Wuhan, China. JAMA Neurol 2020; 77: 683-690.

55 Román GC, Spencer PS, Reis J, et al. The neurology of COVID-19 revisited: a proposal from the Environmental Neurology Specialty Group of the World Federation of Neurology to implement international neurological registries. J Neurol Sci 2020; 414: 116884.

56 Lau HM, Lee EW, Wong CN, et al. The impact of severe acute respiratory syndrome on the physical profile and quality of life. Arch Phys Med Rehabil 2005; 86: 1134-1140.

57 Ahmed H, Patel K, Greenwood DC, et al. Long-term clinical outcomes in survivors of severe acute respiratory syndrome and Middle East respiratory syndrome coronavirus outbreaks after hospitalisation or ICU admission: a systematic review and meta-analysis. J Rehabil Med 2020; 52: jrm00063.

58 International Severe Acute Respiratory and Emerging Infection Consortium. COVID-19 Resources. https://isaric. tghn.org/covid-19-clinical-research-resources/ Date last accessed: July 142020.

59 Petrilli CM, Jones SA, Yang J, et al. Factors associated with hospital admission and critical illness among 5279 people with coronavirus disease 2019 in New York City: prospective cohort study. BMJ 2020; 369: m1966.

60 Belli S, Balbi B, Prince I, et al. Low physical functioning and impaired performace of activities of daily life in COVID-19 patients who survived the hospitalisation. Eur Respir J 2020; 56: 2002096.

61 Lindenauer PK, Stefan MS, Pekow PS, et al. Association between initiation of pulmonary rehabilitation after hospitalization for COPD and 1-year survival among medicare beneficiaries. JAMA 2020; 323: 1813-1823.

62 Mukaino M, Tatemoto T, Kumazawa N, et al. Staying active in isolation: telerehabilitation for individuals with the severe acute respiratory syndrome coronavirus 2 infection. Am J Phys Med Rehabil 2020; 99: 478-479.

63 Wölfel R, Corman VM, Guggemos W, et al. Virological assessment of hospitalized patients with COVID-2019. Nature 2020; 581: 465-469.

64 Hamer M, Kivimäki M, Gale CR, et al. Lifestyle risk factors, inflammatory mechanisms, and COVID-19 hospitalization: a community-based cohort study of 387,109 adults in UK. Brain Behav Immun 2020; 87: 184-187.

65 Lassale C, Gaye B, Hamer M, et al. Ethnic disparities in hospitalisation for COVID-19 in England: the role of socioeconomic factors, mental health, and inflammatory and pro-inflammatory factors in a community-based cohort study. Brain Behav Immun 2020; 88: 44-149.

66 Spitzer KA, Stefan MS, Priya A, et al. A geographic analysis of racial disparities in use of pulmonary rehabilitation after hospitalization for COPD exacerbation. Chest 2020; 157: 1130-1137. 